\title{
A NEARLY EQUAL DISTANT GRID OF ORIENTATIONS FOR QUANTITATIVE TEXTURE ANALYSIS
}

\author{
K. HELMING \\ Institut für Kristallographie und Festkörperphysik, TU Dresden, 01062 Dresden, FRG
}

(Received 18 April 1996)

\begin{abstract}
The construction of a nearly equal distant grid of orientations is presented. Based on that grid texture components can be determined automatically. The obtained set of components can be interpreted as an ensemble of single orientations representing the texture. The grid may be used for the simulation of texture modifying processes. As examples textures of experimental deformed rock salt and aluminium are compared with results of numerical simulations of deformation (Taylor model).
\end{abstract}

KEY WORDS: Orientations, components, ODF-methods, texture modelling.

\section{INTRODUCTION}

Solid state processes in polycrystalline materials are frequently associated with changes of texture caused by changing crystallite orientations. The textures of the initial and final material state can be calculated from diffraction pole figures (PF), e.g. by the component method (Truszkowski et al., 1973; Lücke et al., 1986; Helming and Eschner, 1990). This method can be applied for each crystal symmetry and also for multi-phase materials (Helming, 1993; Helming et al., 1994a). Furthermore texture estimates are possible even in those cases, where the quantity and quality of the measured PFs are limited (Helming et al., 1994b). A component is described by its preferred orientation, the halfwidth and the intensity/volume fraction (Matthies et al., 1987-90). Component orientations and halfwidths can be calculated by applying a non-linear iterative algorithm. For this first estimates are necessary which can be obtained interactively from the graphical representation of the diffraction data.

In this paper the construction of a nearly equal distant grid (NED grid) of orientations is explained (Helming, 1995). The NED grid is characterised by only one parameter, its resolution $\Delta$, which is the maximum distance between two nearest neighbour orientations. For NED orientations and a resolution $\Delta$, a set of texture components can be defined which completely fill the orientation space. The component intensities are determined using a linear fit algorithm. This procedure (NED grid and linear intensity fit) works automatically, i.e. independent of the user.

The set of components based on a NED grid corresponds to a discretisation of the texture. The component intensities may be interpreted as volumes of pseudo crystallites with NED orientations. This texture information can directly be used for numeric simulations of texture modifying processes, assuming that the simulation model does not consider interactions between the pseudo crystallites, like Taylor model (Taylor, 1938) or relaxed constraints (Van Houtte, 1988). 


\section{ORIENTATIONS}

An orientation $\mathrm{g}$ of a crystallite in a sample is described by the rotation $[\omega, \mathrm{n}]$ through an angle $\omega$ around an axis $n=(\vartheta, \varphi)$. g turns the sample coordinate system $K_{A}$ into the crystal coordinate system $K_{B}$. The space of all orientations is called $G$ space. It is symmetrical, since $[-\omega, \mathbf{n}]=[\omega,-\mathbf{n}]$, and compact, because a rotation $[\omega, \mathbf{n}]$ is identical with $[\omega+2 \pi, \mathbf{n}]$. The range of the orientation parameters is therefore given by $0 \leq$ $\omega, \vartheta \leq \pi$ and $0 \leq \varphi<2 \pi$.

Orientations are usually presented by Eulerian angles $\mathrm{g}=\left(\varphi_{1}, \Phi, \varphi_{2}\right)=\left[\varphi_{2}, \mathbf{Z}\right]$ $[\Phi, \mathbf{X}]\left[\varphi_{1}, \mathbf{Z}\right]$ rotating $K_{A}$ into $K_{B}$ by three successive rotations on the topical $\mathbf{Z}, \mathbf{X}$ and $\mathbf{Z}$ axis. Both presentations are related by

$$
\cos \frac{\omega}{2}=\cos \left(\frac{\varphi_{1}-\varphi_{2}}{2}\right) \cos \left(\frac{\Phi}{2}\right), \mathbf{n} \sin \frac{\omega}{2}=\left(\begin{array}{l}
\sin \left(\frac{\varphi_{1}-\varphi_{2}}{2}\right) \sin \left(\frac{\Phi}{2}\right) \\
-\cos \left(\frac{\varphi_{1}-\varphi_{2}}{2}\right) \sin \left(\frac{\Phi}{2}\right) \\
\sin \left(\frac{\varphi_{1}+\varphi_{2}}{2}\right) \sin \left(\frac{\Phi}{2}\right)
\end{array}\right)
$$

The rotation turning the orientation $\mathrm{g}^{\mathrm{c}}=\left[\omega^{\mathrm{c}}, \mathbf{n}^{\mathrm{c}}\right]$ into $\mathrm{g}=[\omega, \mathbf{n}]$ is called orientation difference $\widetilde{\mathrm{g}}^{\mathrm{c}}=\left[\widetilde{\omega}^{\mathrm{c}}, \tilde{\mathrm{n}}^{\mathrm{c}}\right]=\mathrm{g}^{\mathrm{c}} \mathrm{g}^{-1}$ with the orientation distance $\widetilde{\omega}^{\mathrm{c}}$ (Bunge, 1982; Varshalovitc et al., 1975; Matthies et al., 1987-90: Helming, 1995)

$$
\begin{aligned}
\cos \frac{\widetilde{\omega}^{\mathrm{c}}}{2} & =\cos \frac{\omega^{\mathrm{c}}}{2} \cos \frac{\omega}{2}+\mathbf{n}^{\mathrm{c}} \cdot \mathbf{n} \sin \frac{\omega^{\mathrm{c}}}{2} \sin \frac{\omega}{2} \\
& =\cos \frac{\varphi_{1}^{\mathrm{c}}-\varphi_{1}}{2} \cos \frac{\varphi_{2}^{\mathrm{c}}-\varphi_{1}}{2} \cos \frac{\Phi^{\mathrm{c}}-\Phi}{2}-\sin \frac{\varphi_{1}^{\mathrm{c}}-\varphi_{1}}{2} \sin \frac{\varphi_{2}^{\mathrm{c}}-\varphi_{2}}{2} \cos \frac{\Phi^{\mathrm{c}}+\Phi}{2}
\end{aligned}
$$

and the rotation axis $\widetilde{\mathbf{n}}^{\mathrm{c}}$

$$
\tilde{n}^{\mathrm{c}} \sin \frac{\widetilde{\omega}^{\mathrm{c}}}{2}=-\mathbf{n} \sin \frac{\omega}{2} \cos \frac{\omega^{\mathrm{c}}}{2}+\mathbf{n}^{\mathrm{c}} \sin \frac{\omega^{\mathrm{c}}}{2} \cos \frac{\omega}{2}+\mathbf{n} \times \mathbf{n}^{\mathrm{c}} \sin \frac{\omega^{\mathrm{c}}}{2} \sin \frac{\omega}{2}
$$

A distance has to satisfy three demands:

$$
\begin{aligned}
& \widetilde{\omega}\left(g^{1}, g^{2}\right)=\widetilde{\omega}\left(g^{2}, g^{1}\right), \\
& \widetilde{\omega}(g, g)=0, \\
& \widetilde{\omega}\left(g^{1}, g^{2}\right) \leq \widetilde{\omega}\left(g^{1}, g^{3}\right)+\widetilde{\omega}\left(g^{2}, g^{3}\right),
\end{aligned}
$$

which are fulfilled by $\widetilde{\omega}^{c}$. If two different orientations are rotated simultaneously by $\mathrm{g}$, the distance $\widetilde{\omega}$ between them remains unchanged:

$$
\widetilde{\omega}\left(g^{1}, g^{2}\right)=\widetilde{\omega}\left(g^{1}, g g^{2}\right) .
$$

\section{THE NED GRID}

Unlike the presentation with Eulerian angles, where $g=\left(\varphi_{1}, \Phi, \varphi_{2}\right)$ is decomposed into three successive rotations $\left[\varphi_{2}, \mathbf{Z}\right][\Phi, \mathbf{X}]\left[\varphi_{1}, \mathbf{Z}\right]$, now a split into two rotations is introduced by 


$$
\mathbf{g}=[\vartheta, \mathbf{k}]\left[\xi, \mathbf{r}_{\mathbf{A}}\right] \quad \text { with } \quad \vartheta=\angle\left(\mathbf{r}_{\mathbf{A}}, \mathbf{r}_{\mathbf{B}}\right), \quad \mathbf{k}=\frac{\mathbf{r}_{\mathbf{A}} \times \mathbf{r}_{\mathbf{B}}}{\sin \vartheta}
$$

It is based on an arbitrary direction $r$ marked $\mathbf{r}_{A}$ if it is fixed in $K_{A}$ or $\mathbf{r}_{B}$ if it is fixed in $K_{B} \cdot r_{A}$ has the same coordinates in $K_{A}$ as $r_{B}$ in $K_{B}$. The first (right) operator rotates $K_{A}$ into $K^{\prime}$ through $\xi$ on $r_{A}$. The second rotation turns $K^{\prime}$ into $K_{B}$ by $\vartheta$ around the node direction $\mathbf{k}$. $\vartheta$ is the angle (or distance) between $\mathbf{r}_{\mathrm{A}}=\left(\vartheta_{\mathrm{A}}, \varphi_{\mathrm{A}}\right)$ and $\mathbf{r}_{\mathrm{B}}=$ $\left(\vartheta_{\mathrm{B}}, \varphi_{\mathrm{B}}\right)$

$$
\cos \vartheta=\cos \vartheta_{\mathrm{A}} \cos \vartheta_{\mathrm{B}}+\cos \left(\varphi_{\mathrm{B}}-\varphi_{\mathrm{A}}\right) \sin \vartheta_{\mathrm{A}} \sin \vartheta_{\mathrm{B}}
$$

After both operations $r_{A}$ is parallel to $r_{B}$ and $K_{A}$ is identical with $K_{B}$. The orientation distance follows from (2)

$$
\cos \frac{\omega}{2}=\cos \frac{\xi}{2} \cos \frac{\vartheta}{2}
$$

The rotation axis $\mathbf{n}$ lies in a plane with $\left(\mathbf{r}_{\mathbf{A}}+\mathbf{r}_{\mathbf{B}}\right)$ and $\left(\mathbf{r}_{\mathbf{A}} \times \mathbf{r}_{\mathbf{B}}\right)$

$$
2 \sin \frac{\omega}{2} \cos \frac{\vartheta}{2} \mathbf{n}=\sin \frac{\xi}{2}\left(\mathbf{r}_{\mathbf{A}}+\mathbf{r}_{\mathbf{B}}\right)+\cos \frac{\xi}{2}\left(\mathbf{r}_{\mathbf{A}} \times \mathbf{r}_{\mathbf{B}}\right)
$$

in which $\xi$ fixes the direction of $\mathbf{n}$. If $\mathbf{r}=\mathbf{Z}$ the angle $\xi$ is identical with $\sigma=\left(\varphi_{1}\right.$ $\left.+\varphi_{2}\right) / 2$. The angle $\sigma$ was the basic quantity for the introduction of a nearly distortion free ODF presentation (Bunge, 1988; Helming et al., 1988). called $\sigma$-sections (cf. figure 1a). (6) then becomes
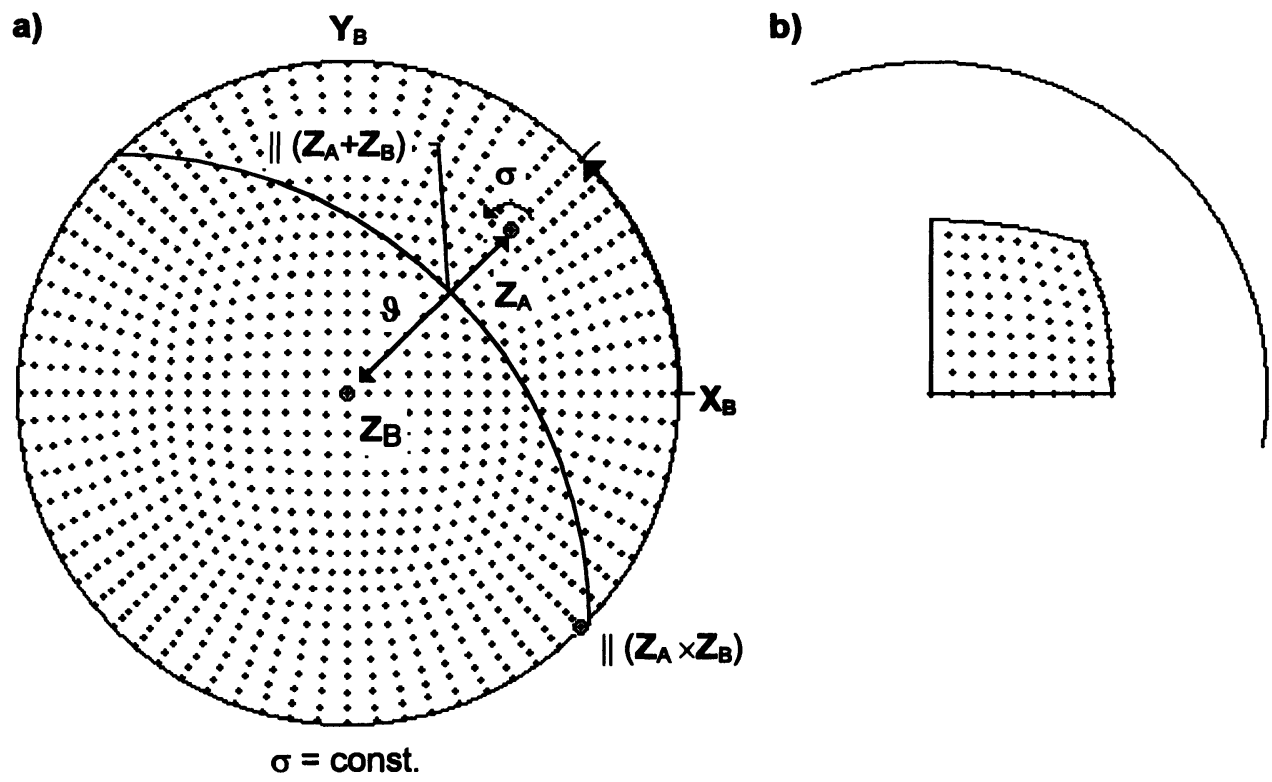

Figure 1 a) Equal area projection of a NED grid with $\Delta=5^{\circ}$ (ref. to $K_{B}$ ). The rotation axis turning $\mathbf{Z}_{\mathbf{A}}$ into $\mathbf{Z}_{\mathbf{B}}$ is coplanar with $\left(\mathbf{Z}_{\mathbf{A}}+\mathbf{Z}_{\mathbf{B}}\right)$ and $\left(\mathbf{Z}_{\mathbf{A}} \times \mathbf{Z}_{\mathbf{B}}\right)$. b) In the case of cubic symmetry only the presented 24th part of a cube surface has to be considered. 


$$
\mathrm{g}=[\beta, \mathbf{k}]\left[\sigma, \mathbf{Z}_{\mathbf{A}}\right] \quad \text { with } \beta=\angle\left(\mathbf{Z}_{\mathbf{A}}, \mathbf{Z}_{\mathbf{B}}\right), \quad \mathrm{k}=\frac{\mathbf{Z}_{\mathbf{A}} \times \mathbf{Z}_{\mathbf{B}}}{\sin \beta}
$$

The orientation distance (cf. (8)) equally depends on the angle $\xi$ and direction distance $\vartheta$. Therefore the three-dimensional G-space can be understood as a combination of a two-dimensional direction space and a one-dimensional angle space.

Let $K_{B}^{1}$ and $K_{B}^{2}$ be the coordinate systems of two crystallites different from $K_{A}$. The coordinates of their $Z$-axes referred to $K_{A}$ are $Z_{1}=\left(\vartheta_{1}, \varphi_{1}\right)$ and $Z_{2}=\left(\vartheta_{2}, \varphi_{2}\right)$ If $Z_{1} \|$ $Z_{2}$ the distance $\widetilde{\omega}_{12}\left(g_{1}, g_{2}\right)$ between $g_{1}$ and $g_{2}$ reads $\widetilde{\omega}_{12}=\sigma_{1}-\sigma_{2}$. For $\sigma_{1}=\sigma_{2}$ the distance $\widetilde{\omega}_{12}\left(\mathrm{~g}_{1}, \mathrm{~g}_{2}\right)$ is given by

$$
\cos \frac{\widetilde{\omega}_{12}}{2}=\cos \frac{\vartheta_{1}}{2} \cos \frac{\vartheta_{2}}{2}+\cos \left(\varphi_{2}-\varphi_{1}\right) \sin \frac{\vartheta_{1}}{2} \sin \frac{\vartheta_{2}}{2}
$$

$\widetilde{\omega}_{12}\left(\mathrm{~g}_{1}, \mathrm{~g}_{2}\right)$ is identical with the distance $\widetilde{\omega}(\mathrm{e}, \widetilde{\mathrm{g}})$ between $\mathrm{e}=[0, \mathrm{n}]$ and $\widetilde{\mathrm{g}}=\mathrm{g}_{1}{ }^{-1} \mathrm{~g}_{2}$. where $K_{B}^{1}$, equals $K_{A}$ (cf. (5)). For $\widetilde{\xi}=0\left(Z_{1}\right.$ not parallel to $\left.Z_{2}\right)$. we have

$$
\widetilde{\omega}=\widetilde{\vartheta}=\angle\left(\mathbf{Z}_{\mathbf{A}}, \tilde{\mathbf{Z}}\right)=\angle\left(\mathbf{Z}_{1}, \mathbf{Z}_{2}\right)
$$

To construct a NED grid of orientations where the distances to nearest neighbours and its numbers are nearly constant for each orientation, one firstly needs a nearly equal-distant grid of directions. The equal area projection of that kind of direction grid (ref. to $K_{B}$ ) is shown in figure 1a. Its construction is based on a cube (Helming. 1995) with the edges parallel to the coordinate axes of $\mathrm{K}_{\mathrm{B}}$. Cube and pole sphere are concentric. On the cube face for $\mathrm{x}=$ const the directions shown are given by cartesian coordinates

$\mathrm{r}_{\mathrm{ij}}=\left(\begin{array}{l}\mathrm{x} \\ \mathrm{y} \\ \mathrm{z}\end{array}\right)=\frac{1}{\mathrm{~N}_{\mathrm{ij}}}\left(\begin{array}{c}1 \\ \tan (\mathrm{i} \Delta) \\ \tan (\mathrm{j} \Delta)\end{array}\right) \quad$ with $\quad \mathrm{N}_{\mathrm{ij}}=\sqrt{1+\tan ^{2}(\mathrm{i} \Delta)+\tan ^{2}(\mathrm{j} \Delta)}$

and $-\tau<\mathrm{i}, \mathrm{j} \leq \mathrm{z}$.

where $\tau=90^{\circ} / \Delta$ must be an integer, even number. $\Delta$ is the maximum angle between nearest neighbour directions. It should be a divisor of $45^{\circ}$. In the same manner the directions for the remaining five cube faces can be built. The special arrangement of directions in the cube grid allows two-dimensional interpolations.

Two steps are necessary to create a NED grid of orientations. At first each direction in figure la should be identified as a $\mathbf{Z}_{\mathrm{A}}$-axis (ref. to $\mathrm{K}_{\mathrm{B}}$ ) Secondly orientations with the same $\mathbf{Z}_{\mathbf{A}}$-axis but different $\sigma$ (cf. (10)) are distinguished by different $\sigma$-sections (cf. figure 1a). The partition of $\sigma$ (cf. (7)) is $\sigma=0, \Delta, 2 \Delta, \ldots 360^{\circ}-\Delta$ with $\Delta$ of the direction grid. Without any symmetry the total number $\mathrm{T}$ of single orientations $\mathrm{g}^{\mathrm{t}}(0$ $\leq \mathrm{t}<\mathrm{T})$ is

$$
\mathrm{T}=4 \tau\left(6 \tau^{2}+2\right)
$$

since the number of $\sigma$-values for a $Z_{\mathrm{A}}$-axis is $4 \tau$ and the number of directions on the cube is $6 \tau^{2}+2$ (six faces with $\tau^{2}$ points and two remaining edges). The NED grid is completely determined by the angle $\Delta$ called resolution. The resolution $\Delta$ characterises the maximum distance between nearest neighbour $\mathrm{g}^{\mathrm{t}}$. For $\Delta=5^{\circ}(\tau=18)$ only 140112 orientations are necessary unlike a $5^{\circ}$-partition based on the Eulerian angles $\varphi_{1}, \Phi, \varphi_{2}$ with $\left(360^{\circ} / 5^{\circ}\right)^{2}\left(180^{\circ} / 5^{\circ}+1\right)=181476$ orientations. 
Since $K_{B}$ and not $K_{A}$ is the reference system (figure 1a), an existing crystal symmetry can be used to reduce $\mathrm{T}$ in a simple manner. In the case of cubic symmetry one gets $\mathrm{T}=5838\left(\Delta=5^{\circ}\right)$, because only the 24th part of a cube surface (presented in figure $1 \mathrm{~b})$ has to be considered. If a $\mathbf{Z}_{\mathrm{A}}$-axis is parallel to an $\mathbf{n}$-fold symmetry axis of the crystallite the range of $\sigma$ is reduced to $0<\sigma \leq 360 / \mathrm{n}$,

\section{TEXTURE COMPONENTS}

The texture of a crystalline phase in a polycrystalline material is quantitatively given by the orientation density function (ODF):

$$
f(g)=\frac{8 \pi^{2}}{V} \frac{d V(g)}{d g} \geq 0
$$

describing the volume fraction of all crystallites for each orientation $g \in d g$ (Bunge 1982; Matthies et al., 1987-90).

Pole figures (PF) which can be measured in a diffraction experiment can be understood as two-dimensional projections of the three-dimensional ODF

$$
\tilde{P}_{h}(y)=\frac{1}{2}\left[P_{h}(y)+P_{-h}(y)\right]=\frac{1}{2} \int_{0}^{\pi}[f([(\varphi,+y \| h])+f([\varphi,-y \| h])] d \varphi .
$$

Here $\mathrm{g}_{\varphi}=[\varphi, \mathrm{y} \| \mathrm{h}]$ with $0 \leq \varphi \leq \pi$ describes all orientations with the lattice plane normal $h$ (ref. to $K_{B}$ ) parallel to the scattering vector $y$ (ref. to $K_{A}$ ). $y$ is called sample direction. Hence PFs describe density distributions of crystal directions $h$ (ref. to $K_{A}$ ). Because of Friedel's law measured PFs $\widetilde{\mathbf{P}}_{\mathrm{h}}(\mathbf{y})$ are always centrosymmetric. Starting from a given ODF the PFs for arbitrary crystal directions $\mathbf{h}$ can be calculated. Often the $\mathrm{PF}$ of a special $\mathrm{h}$ is more expressive than the ODF-presentations by $\varphi_{1}, \varphi_{2}$ - or $\sigma$-sections.

The ODF-approximation by means of texture components

$$
\begin{aligned}
& \mathrm{f}(\mathrm{g})=\mathrm{F}+\sum_{\mathrm{c}} \mathrm{I}^{\mathrm{c}} \mathrm{f}^{\mathrm{c}}(\mathrm{g}) \\
& \text { with } \mathrm{F}+\sum_{\mathrm{c}} \mathrm{I}^{\mathrm{c}}=1 \text { and } \oint_{G} \mathrm{f}^{\mathrm{c}}(\mathrm{g}) \mathrm{dg}=1
\end{aligned}
$$

(Truszkovski et al., 1973; Bunge, 1982; Matthies et al., 1987-90) is based on the earlier concepts of ideal orientations (Wassermann, 1939). A component is described by a model function $\mathrm{f}^{\mathrm{c}}(\mathrm{g})$, which is locally restricted in the G-space. It has a maximum at a preferred orientation $\mathrm{g}^{\mathrm{c}}$ and decreases with increasing orientation distance $\widetilde{\omega}^{\mathrm{c}}$ (cf. (2)). The intensity $\mathrm{I}^{\mathrm{c}}$ describes the volume fraction of all crystallites belonging to the component c. The quantity $F$ gives the volume fraction of the crystallites, which are randomly oriented in the sample. It may be understood as the intensity of the only global component used in the model, which is given by $\mathrm{f}^{\mathrm{c}}(\mathrm{g})=1$ for each $\mathrm{g} \in \mathrm{G}$. The Gaussian model function (Matthies et al., 1987-90) used here is given by

$$
\begin{aligned}
& f^{c}(g)=N^{c} e^{S^{c} \cos \varpi^{c}} \\
& \text { with } S^{c}=\frac{\ln 2}{1-\cos \left(b^{c} / 2\right)} \text { and } N^{c}=\frac{1}{I_{0}\left(S^{c}\right)-I_{1}\left(S^{c}\right)}
\end{aligned}
$$


where $I_{1}(x)$ are generalised Bessel functions. The value $b^{c}$ is the halfwidth and can be interpreted as the mean diameter of a spherical component in the orientation space. The corresponding PFs are given by closed analytical expressions

$$
\mathbf{P}_{\mathbf{h}}^{\mathrm{c}}(\mathbf{y})=\mathbf{N}^{\mathrm{c}} \mathrm{e}^{\mathrm{s}^{\mathrm{c}} \sin \left(v^{\mathrm{c}} / 2\right)} \mathrm{I}_{0}\left(\mathbf{S}^{\mathrm{c}} \cos \left(v^{\mathrm{c}} / 2\right)\right) \text { with } \cos v^{\mathrm{c}}=\mathbf{h}\left(\mathrm{g}^{\mathrm{c}} \mathbf{y}\right) .
$$

\section{AUTOMATIC COMPONENT METHOD}

The components (cf. (18)) describing $\mathrm{f}(\mathrm{g})$ can be determined by the best fit of the experimental data $\widetilde{\mathrm{P}}_{\mathrm{h}_{\mathrm{i}}}\left(\mathbf{y}_{\mathbf{r}}\right) / \mathrm{N}_{\mathrm{h}_{\mathrm{i}}}$ with the recalculated pole figures $\sum_{c} I^{c} \widetilde{\mathrm{P}}_{\mathbf{h}_{\mathrm{i}}}^{\mathrm{M}}\left(\mathrm{g}^{\mathrm{c}}, \mathbf{b}^{\mathrm{c}}, \mathbf{y}_{\mathbf{r}}\right)$.

The calculation of the model pole figures $\widetilde{\mathrm{P}}_{\mathrm{h}_{\mathrm{i}}}^{M}$ follows from (16) and (19). The index $\mathrm{r}$ marks the measured sample directions $\mathbf{y}_{\mathbf{r}}$.

The component parameters $I^{c}, g^{c}$ and $b^{c}$ and the normalisatior. $N_{h_{i}}$ of the PFs are obtained by solving the least squares problem ( $w_{\text {ir }}$ are weight factors)

$$
\sum_{i, r} w_{i r}\left[\widetilde{P}_{h_{i}}\left(\mathbf{y}_{\mathbf{r}}\right) / N_{h_{i}}-\sum_{c} I^{c} \widetilde{P}_{h_{i}}^{M}\left(g^{c}, b^{c}, \mathbf{y}_{r}\right)\right]^{2} \Rightarrow \text { Min }
$$

Usually the parameters $g^{c}$ and $b^{c}$ must be calculated by a non-linear algorithm. First estimates are necessary, which may be obtained interactively from the graphical representation of the difference pole figures

$$
\Delta_{h_{i}}\left(\mathbf{y}_{\mathbf{r}}\right)=\widetilde{P}_{\mathbf{h}_{i}}\left(\mathbf{y}_{\mathbf{r}}\right)-\sum_{c} I^{c} \widetilde{P}_{h_{i}}^{M}\left(g^{c}, b^{c}, \mathbf{y}_{\mathbf{r}}\right)
$$

on a PC monitor. This procedure is explained in more detail in (Helming and Eschner, 1990; Helming, 1993). Another possibility to define $\mathrm{g}^{\mathrm{c}}$ and $\mathrm{b}^{\mathrm{c}}$ is based on the NED grid described above. The preferred orientations $\mathrm{g}^{\mathrm{c}}$ are given by the $\mathrm{T}$ single orientations $\mathrm{g}^{\mathrm{t}}$ of the NED grid. The values $b^{\mathrm{c}}$ for all components should be equal to the resolution $\Delta$. Only the intensities $I^{c}$ must be calculated applying the least squares fit (20). This only requires a linear algorithm (I-fit) that can be performed automatically.

If all intensities $I^{c}$ are set to $1 / T$ an isotropic ODF with $f(g)=1$ is expected. In figure 2a the corresponding (111), (200) and (220) PFs are shown, produced by 218 components for $\Delta=15^{\circ}$ (cubic crystal symmetry). Considering that the construction is based only on geometrical principles, the PFs show the expected isotropy. To improve the ODF the intensities Ic were refined fitting PFs with $\widetilde{P}_{h_{i}}\left(y_{r}\right)=1$. The recalculated PFs are shown in figure $2 \mathrm{~b}$. The visible deviations are caused by the geometric assumptions of the NED grid and the behaviour of the distribution (18).

To describe an arbitrary ODF with NED grid components the I-fit has to be performed with the corresponding PFs. To reduce a loss of texture information caused by the component model, the resolution $\Delta$ should be as small as possible (but not smaller than the resolution of the measured pole figures). This is explained in figure 3 where the texture of rock salt (natural state, PFs measured with neutrons, Scheffzuik 1995) was approximated by means of three NED grids with $\Delta=45^{\circ}, 15^{\circ}, 11.25^{\circ}(\mathrm{T}=9$. 218,515 respectively).

If the I-fit results in some components with negative intensities, the solution should be considered as a first estimate of an iterative algorithm. Negative intensities may be caused e.g. by errors of measurement. The corresponding components should be deleted before the next iteration step, i.e. before repeating the I-fit. In figure 4a the 
(111)

a)

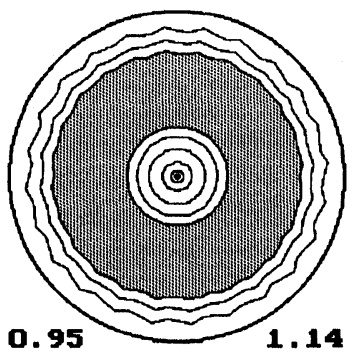

b)

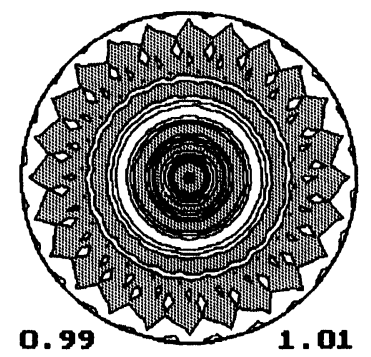

(200)
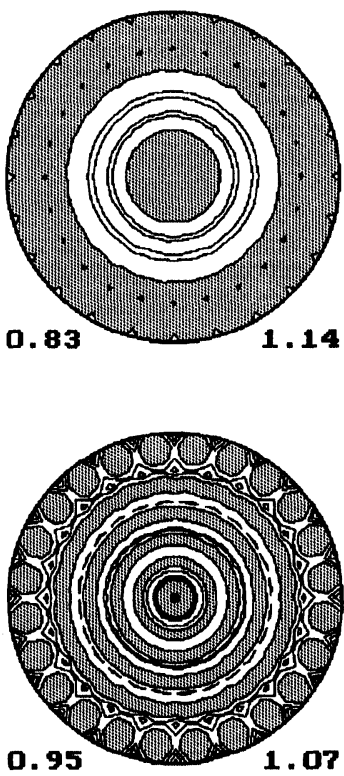

(220)
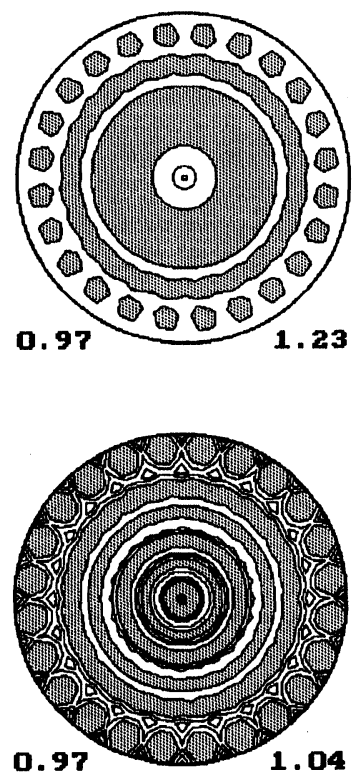

Figure 2 (111), (200) and (220) PFs generated by 218 components for $\Delta=15^{\circ}$ (cubic crystal symmetry) with a) $I^{c}=1 / 218$ and $\left.b\right) I^{c}$ calculated by the I-fit. Minimum and maximum of PF-values are given, ranges with $\widetilde{\mathrm{P}}_{\mathbf{h}_{\mathbf{i}}}\left(\mathbf{y}_{\mathbf{r}}\right)<\mathrm{I}$ are shaded.

(111), (200) and (220) PFs of a casted $\mathrm{Al}$ composite (Mg $<1,5 \%$, measured with $\mathrm{X}$ rays) are shown (Fels, 1996). Because of the large grain size $(\approx 100 \mu \mathrm{m})$ they are affected by a poor statistics. The component description is based on two NED grids with $\Delta=15^{\circ}$ and $11.25^{\circ}$. As shown in figure 5 the number $\mathrm{Tm}$ of remaining positive components decreases with increasing iteration step $\mathrm{m}$ down to a limit of $\mathrm{T}_{4}=60$ and $\mathrm{T}_{5}=106$, respectively. The recalculated PFs of the final solution $\left(\mathrm{m}=5, \Delta=11.95^{\circ}\right)$ are presented in figure $4 \mathrm{~b}$. The same material has been extruded (rectangular crosssection). Using NED components with $\Delta=15^{\circ}(\mathrm{T}=218)$ the I-fit yields 37 components after five steps. Figure 6 shows the measured and recalculated PFs

\section{TEXTURE SIMULATIONS}

The modelling of processes in polycrystals is often based on handling large numbers of independent single grain orientations. Their choice has to be suitable to represent the texture of the materials initial state. For $\Delta \rightarrow 0$ the NED components correspond to a discretisation of the ODF, i.e. the component intensities may be interpreted as volumes of pseudo crystallites with NED orientations. The NED crystallites generated by means of the automatic component method from PFs of natural rock salt (figure 3) and casted $\mathrm{Al}$ (figure 4) have been taken as initial states for the simulation of 
(111)

a)

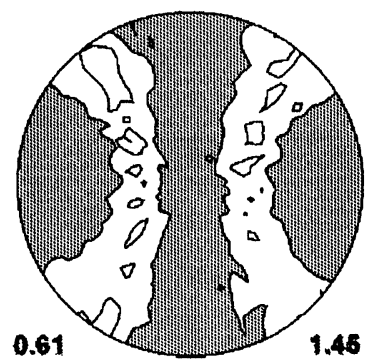

b)

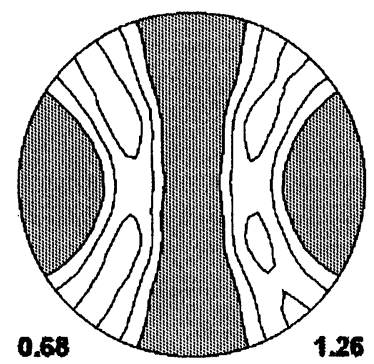

c)

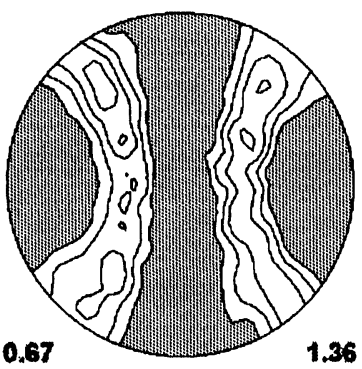

d)

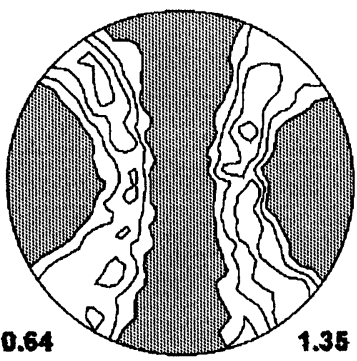

(200)
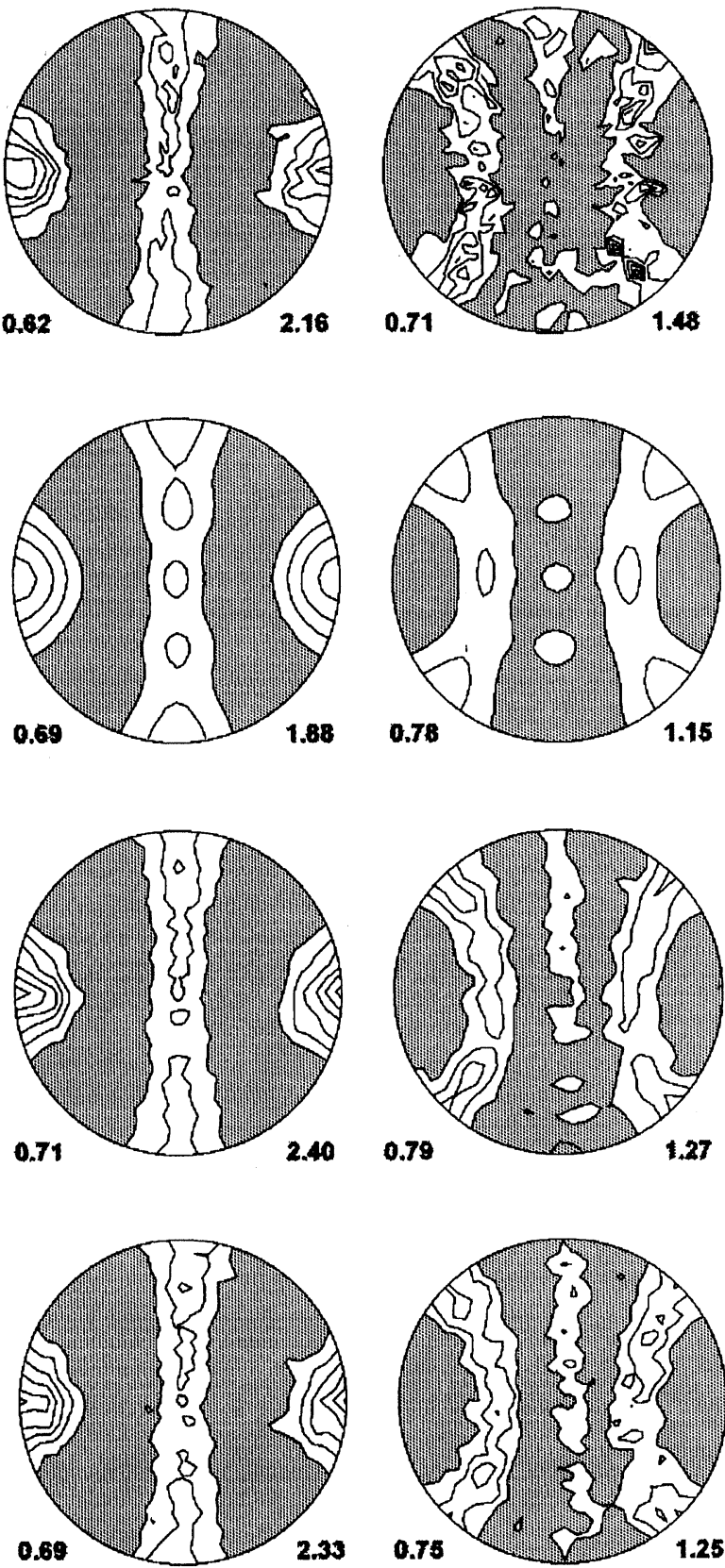

(220)

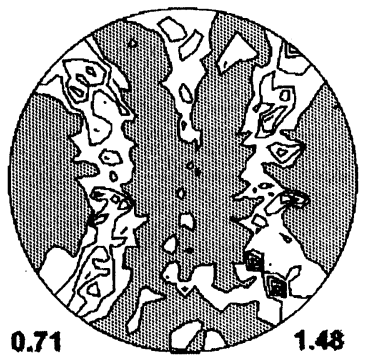

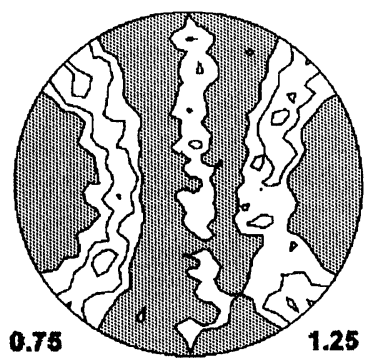

Figure 3 PFs of rock salt measured with neutrons: a) natural state, from Scheffzük et al., b)-d) approximations by means of the NED grids with $\Delta=45^{\circ}, 15^{\circ}, 11.25^{\circ}(\mathrm{T}=9,218,515$ respectively). 
(111)

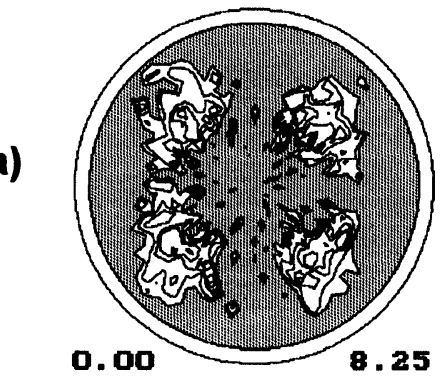

0. 00

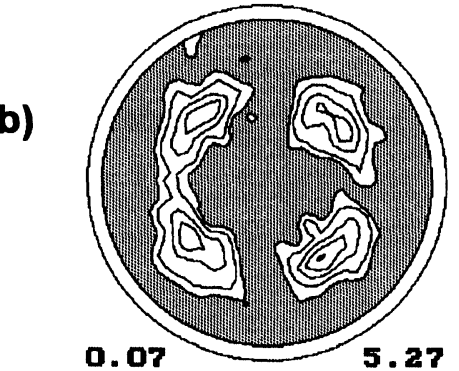

(200)
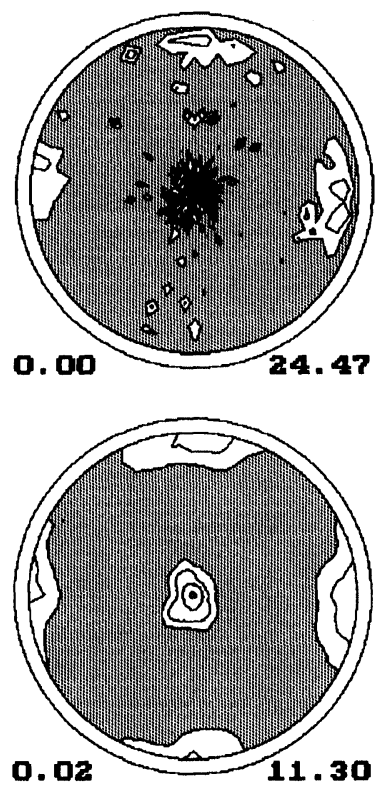

(220)
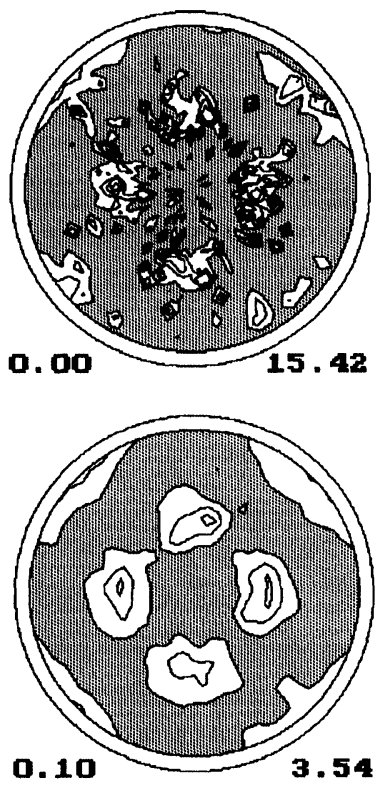

Figure 4 a) (111), (200) and (220) PFs of a cast $\mathrm{Al}$ composite ( $\mathrm{Mg}<1,5 \%$, measured with X-rays) from Fels (1969). Because of the large grain size $(\approx 100 \mu \mathrm{m})$ they are affected by a poor statistics. b) The texture description by 106 components is based on a NED grid with $\Delta=11,25^{\circ}$ and an iterative reduction (I-fit, five steps) of the component number (cf. tab. 1).

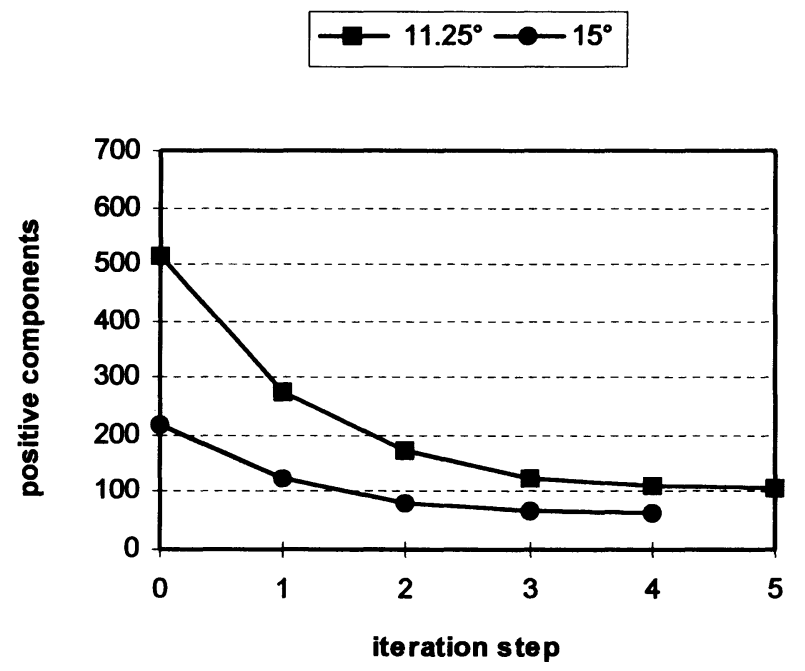

Figure 5 Number of remaining positive NED components with $\Delta=15^{\circ}$ and $11.25^{\circ}$ versus number of iteration steps. The recalculated PFs of the final solution (five steps $\Delta=11.25^{\circ}$ ) are shown in figure $4 \mathrm{~b}$. 
(111)

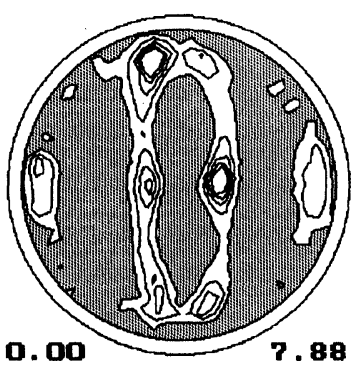

b)

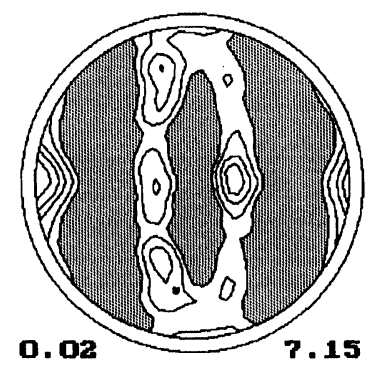

(200)
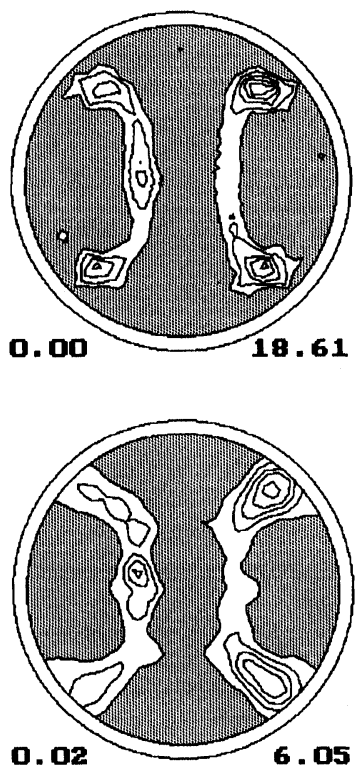

(220)
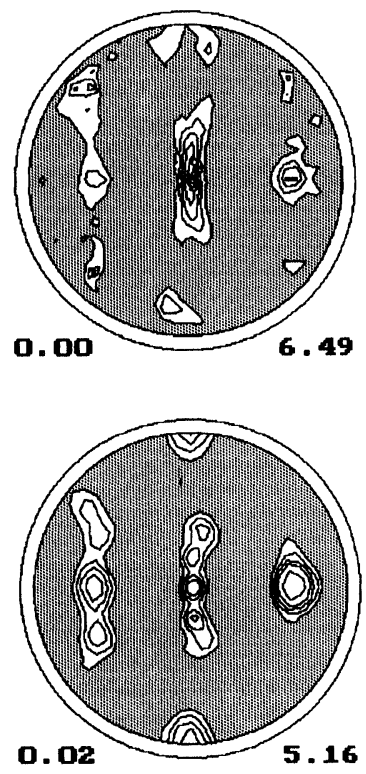

Figure 6 a) Measured and b) recalculated PFs of extruded $\mathrm{Al}$ (rectangular crosssection) Using NED components with $\Delta=15^{\circ}(\mathrm{T}=218)$ the I-fit yields 37 components after five steps.

deformation. Compression and extrusion (round cross-section) was simulated using the FC Taylor model (PC program by van Houtte, 1988). $\mathbf{Z}_{\mathbf{A}}$ was chosen parallel to the compression/extrusion-axis. The initial state PFs of rock salt show a (200) $\perp \mathbf{Z}_{\mathbf{A}}$-fibre. those of Lasted $\mathrm{Al}$ a cube texture. In the case of rock salt the glide systems $\{100\}<110>$ and $\{110\}<110>$ with equal critical resolved shear stresses and a total strain of $20 \%$ were used as input parameters. In additiopn to a (220)\| $\mathbf{Z}_{\mathbf{A}}$ fibre induced by the deformation a (200) $\perp \mathbf{Z}_{\mathbf{A}}$ fibre is visible in the PFs (cf. figure 7a). The (200) $\perp \mathbf{Z}_{\mathbf{A}}$ fibre is a remainder of the initial texture. The result corresponds to the PFs of the experimental deformed sample (20\% strain; Scheffzük, 1995) shown in figure $7 \mathrm{~b}$.

In the case of the Al sample the simulation was carried out with glide systems

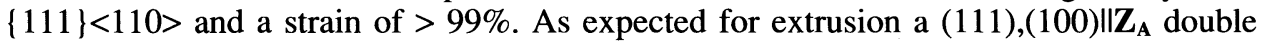
fibre texture is obtained. The axial symmetry is disturbed by some spherical components. They should be meta-stable orientations occurring at the transition from the initial cube texture to the final double fibre texture. The corresponding PFs (figure 8a) are compared with the incompletely measured PFs of the experimentally deformed sample ( $80 \%$ strain; Fels, 1996) shown in figure $8 \mathrm{~b}$. 
(111)

a)

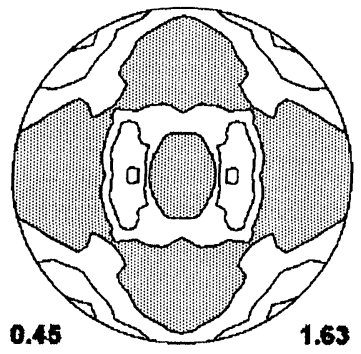

b)

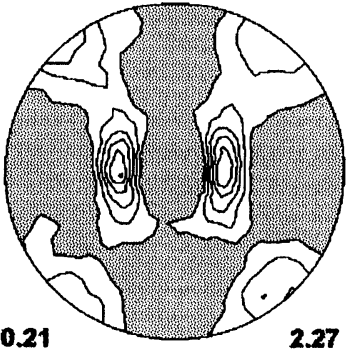

(200)
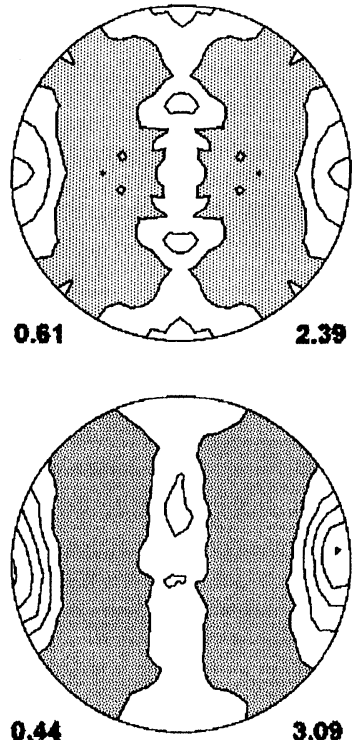

(220)
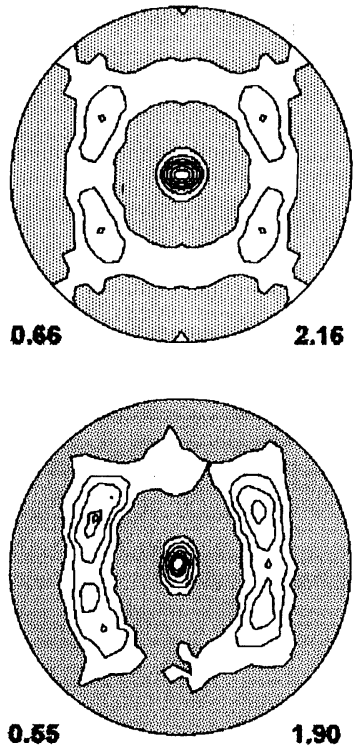

Figure 7 PFs of compressed rock salt (20\% strain): a) Taylor-simuiated b) experimentally deformed (Scheffzuik, 1995). The initial texture is shown in figure 3a.

(111)

a)

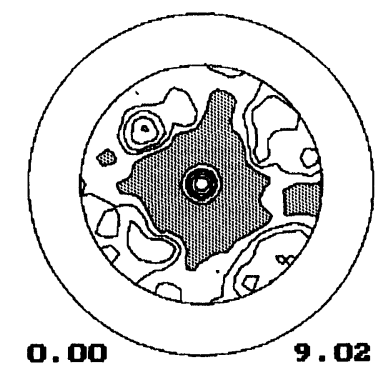

b)

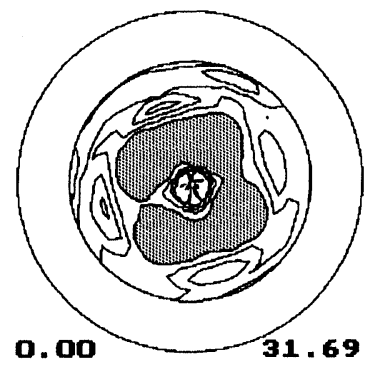

(200)
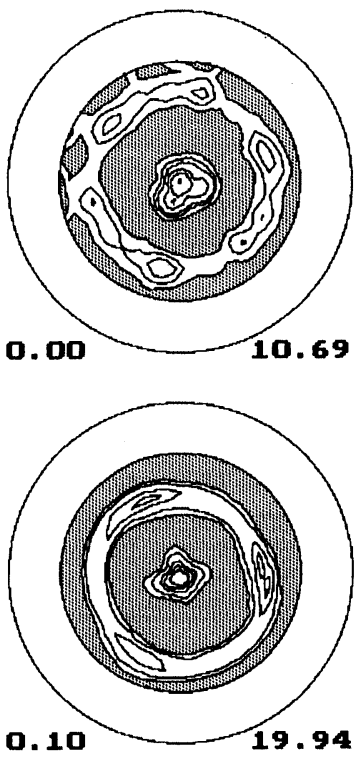

(220)
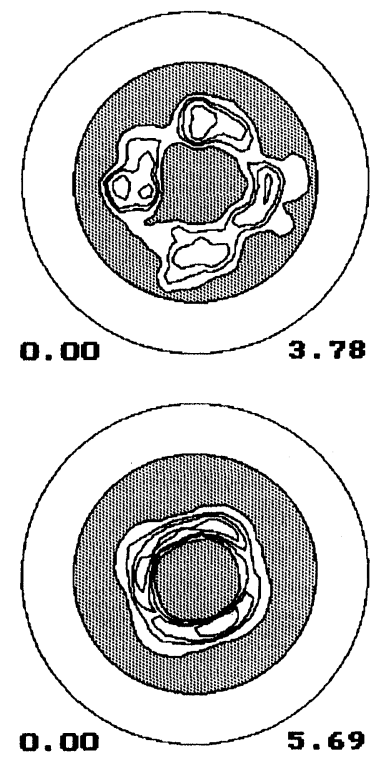

Figure $8 \mathrm{PFs}$ of $\mathrm{Al}$ extruded through a round die: a) Taylor simulated (>99\% strain) b) experimentally deformed $(80 \%$ deformation; Fels, 1996). The initial texture is shown in figure $4 \mathrm{a}$. 
of the extensive interactive search for preferred orientations. On the other hand the disadvantage of a large number of (initial) NED components must be accepted. For some applications like Taylor modelling the number of NED components should be as large as possible $(\Delta \rightarrow 0)$.

The used PC-version allows to calculate with a maximum of 1000 components. This is sufficient for the presented examples $\left(\Delta \approx 10^{\circ}\right.$, cubic crystal symmetry). For smaller resolutions or lower crystal symmetry improvements of soft- and hardware are required.

Based on the NED grid, graphical presentations of ODF for arbitrary directions of projections (not limited to $\mathrm{Z}_{\mathrm{A}}$ axis) can be imagined. These presentations will reflect the orientation distance adequately like o-sections.

\section{Acknowledgement}

The author wishes to thank Ch. Schefzük and B. Fels for graciously supplying the measured pole figures. Particular thanks are due to R. Tamm and W. Skrotzki who have reviewed and commented this manuscript.

\section{Literature}

Bunge, H. J. (1982). Texture Analysis in Materials Science, Butterworth London.

Bunge, H. J. (1988). In: J. S. Kallend und G. Gottstein (Hrsg.): Proc. $8^{\text {th }}$ Int. Conf. Text. Mat. (ICOTOM 8). Warrendale, PA TMS, 69-78.

Fels B. (1996). Textur- und Mikrostrukturuntersuchungen an stranggepre $\beta$ ten Aluminum, Diplom Thesis, TU Dresden.

Helming, K., S. Matthies and G. Vinel (1988). In J. S. Kallend und G. Gottstein (Hrsg.): Proc. Eighth Int. Conf. Text. Mat. (ICOTOM 8). Warrendale, PA TMS, 55-60.

Helming, K. and T. Eschner (1990). Cryst. Res. Technol., 25, K203-K208.

Helming, K. (1994). Physics of the Solid Earth, engl. translation: Vol. 29, No. 6, 523-532; russ. edition (1993), 73-82.

Helming, K., H. -R. Wenk, C. S. Choi and W. Schäfer (1994a). In: H. J. Bunge, S. Siegesmund. W Skrotzki, K. Weber (Eds): Textures of Geological Materials. DGM Press, 303-326.

Helming, K., R. A. Schwarzer, B. Rauschenbach, S. Geier, B. Leiss, H. Wenk, K. Ullemeier and J. Heinitz (1994b). Z. Metalikd., 8, 545-553.

Helming, K. (1995). Texturapproximation durch Modellkomponenten. Habilitation Thesis. TU Clausthal.

Lücke, K., J. Pospiech, J. Jura and J. Hirsch (1986). Z. Metalikd., 77, 312-321.

Matthies, S., G. W. Vinel and K. Helming (1987-90). Standard Distributions in Texture Analysis. Vol I-III, Akademie-Verlag Berlin.

Schefzük, Ch., A. Frischbutter and P. Bankwitz (1995). Scientific technical report STR95/19. GFZ Potzdam .

Truszkowski, W., J. Pospiech, J. Jura und B. Major (1973). In Proc. 3è Colloque Européen sur les Textures de Deformation et de Recristallisation des Metaux et leurs Applications Industrielles (Ed. R. Penelle), Pont-a-Mousson (1973), 235-257.

Taylor, G. I. (1938). J. Inst. Met., 62, 307.

Van Houtte, P. (1988). Textures and Microstructures, 8\&9, 313-350.

Varshalovitc, D. A., A. N. Moskalev and V. K. Chersonskij (1975). Kvantovaja teorija uglowo momenta. Verlag Nauka, Leningrad.

Wassermann, G. (1939). Texturen metallischer Werkstoffe, Springer Verlag; G. Wassermann and J. Grewen (1962). Texturen metallischer Werkstoffe, Springer Verlag. 